\title{
Meet the editors
}

\author{
This month at Nature Computational Science, we welcome a new member to our team. We would like to take this \\ opportunity to briefly describe who we are and what our main responsibilities entail.
}

ooking back, it is hard to believe that when Nature Computational Science first opened for submissions in April 2020, the editorial team consisted of only a single editor, in addition to help from an editor from Nature Machine Intelligence. The team would soon grow to a total of three editors within the span of about six months, and this month, in order to support the growth of the journal and the diversity of scientific disciplines included in its scope, it is with great excitement that we welcome a fourth member to our team.

But who are we and what do we do?

As we described in a previous Editorial, our team consists of full-time professional editors. This means that we are entirely dedicated to the editorial process at the journal and that we are not associated with any university or research laboratory/ institution, in order to avoid potential biases when assessing manuscripts. Our decisions are solely based on the scientific content of submitted manuscripts, rather than on the authors' names and institutions.

Because our journal has a strong multidisciplinary nature, covering a multitude of domain areas, our team comprises diverse scientific backgrounds. Each of our editors holds a $\mathrm{PhD}$ in a discipline of relevance to the journal, and their unique expertise dictates the scientific areas that they are each primarily responsible for managing. Fernando Chirigati has a $\mathrm{PhD}$ in computer science, having worked on large-scale data analytics and data mining, and takes care of content from computer science, applied mathematics, and urban science. Ananya Rastogi is trained in systems immunology and has worked on different mathematical biology research projects, and is mainly responsible for computational biology and computational social sciences manuscripts. The materials science and physics-related content of the journal is handled by Jie Pan, who has an extensive background in these areas, particularly in regards to computational materials design. Computational chemistry will be handled by the most recent member of our team, Kaitlin McCardle, who studied molecular electrocatalysts using various computational techniques in her doctoral program.

While editors primarily handle manuscripts that are closer to their research expertise, in certain situations we may need to take care of content that does not precisely match our professional backgrounds. For instance, our journal certainly considers work from areas that are not mentioned in the above paragraph, and in this case, the chief editor identifies which editor has the knowledge that is most compatible with the topic. In addition, different areas have different submission rates that vary over time, so depending on the current editorial workload, manuscripts from a specific area might need to be distributed among all of the editors. In these cases, even though our backgrounds may not perfectly align with a manuscript, we are well-versed in assessing scientific research and results in general, and are equipped to use our best editorial judgment in order to make an informed decision. It is worth noting that, as an important editorial practice of our journal, a manuscript is always discussed by at least two of our editors before an editorial decision is made, helping to ensure that all pertinent details from the manuscript are considered.

Handling the primary research papers that are submitted to the journal (from assessing their suitability to the journal to overseeing the review and publication process) is not our only responsibility. One of our main tasks for the journal is to also work on and handle non-primary research content (for instance, Reviews, Perspectives, and Comments): these are mostly commissioned by us to provide a forum for researchers to discuss and comment on technical and non-technical issues that are highly relevant to the computational science community. For both primary and non-primary content, we perform careful editing to make sure that the science is communicated to our readers in the best possible way. We also keep abreast of recent developments by attending conferences and visiting research labs. Finally, we organize events and workshops for the research community, and work on collections and focused issues, sometimes across different journals of the Nature Portfolio family, on topics that are of relevance to our readership.

Needless to say, if we are successful in what we do, it is because you, authors and readers of Nature Computational Science, have put your trust in us and our editorial process. We would like to take this opportunity to thank you for your trust, and we hope to meet you (hopefully in person) in a future conference or event sometime soon!

Published online: 22 September 2021 https://doi.org/10.1038/s43588-021-00137-5 